\title{
Brownian Motion in a Convex Ring and Quasi-Concavity
}

\section{Christer Borell}

Chalmers University of Technology, S-41296 Göteborg, Sweden

\begin{abstract}
Let $X$ be the Brownian motion in $\mathbb{R}^{n}$ and denote by $\tau_{M}$ the first hitting time of $M \cong \mathbb{R}^{n}$. Given convex sets $K \cong L \leqq \mathbb{R}^{n}$ we prove that all the level sets

$$
\left\{(x, t) \in \mathbb{R}^{n} \times\left[0,+\infty\left[; P_{x}\left[\tau_{K} \leqq t \wedge \tau_{L^{c}}\right] \geqq \lambda\right\}, \lambda \in \mathbb{R},\right.\right.
$$
\end{abstract}

are convex.

\section{Introduction}

The background of the present paper is a very beautiful theorem of Gabriel $[3,4]$ and Lewis [5] stating that the equilibrium potential of a convex body in $\mathbb{R}^{n}$ relative to a surrounding convex body is quasi-concave. Below we will show the same property for the solution of the corresponding heat conduction problem with zero initial data. Here recall that a real-valued function $f$ defined on a convex set is said to be quasi-concave if all the level sets $\{f \geqq \lambda\}, \lambda \in \mathbb{R}$, are convex.

Throughout, $X$ denotes the Brownian motion in $\mathbb{R}^{n}$ and, for each $M \cong \mathbb{R}^{n}, \tau_{M}$ stands for the first hitting time of $M$, that is, $\tau_{M}=\inf \{t>0 ; X(t) \in M\}$.

Theorem 1.1. Suppose $K, L \subseteq \mathbb{R}^{n}$ are convex sets such that $K \cong L$. Then the function

$$
w(x, t)=P_{x}\left[\tau_{K} \leqq t \wedge \tau_{L^{c}}\right], \quad(x, t) \in \mathbb{R}^{n} \times[0,+\infty[,
$$

is quasi-concave.

Here, for short, $L^{c}$ means $\mathbb{R}^{n} \backslash L$.

To prove Theorem 1.1 there is no loss of generality to assume that (i) $K$ is a convex body in $\mathbb{R}^{n}$, (ii) $L$ is the interior of a convex body in $\mathbb{R}^{n}$, and (iii) $d\left(K, L^{c}\right)>0$. In what follows, we always assume (i)-(iii) are fulfilled. Then, in particular,

$$
\left\{\begin{array}{lll}
\Delta w=2 w_{t}^{\prime} & \text { in } & (L \backslash K) \times] 0,+\infty[ \\
w=0 & \text { on } & \{(L \backslash K) \times\{0\}\} \cup\{\partial L \times[0,+\infty[\} \\
w=1 & \text { on } & \partial K \times[0,+\infty[
\end{array}\right.
$$


and, hence, $w(x,+\infty)$ is the equilibrium potential of $K$ relative to $L$ (see e.g. Friedman [2]).

The proof of Theorem 1.1 is divided into three steps. In the first step we use the isoperimetric inequality of Brownian motion to obtain a certain differential inequality, which is basic for the subsequent arguments. Theorem 1.1 then follows from Step 1 exploiting the same main line of reasoning as in the time-stationary case (Step 3). There is just one new difficulty, namely to handle the discontinuity points of $w$ (Step 2).

\section{Step 1: A Differential Inequality}

Suppose $(x, t) \in(L \backslash K) \times] 0,+\infty[$ and $y \in K$ are fixed.

We claim that

$$
(y-x) \cdot \nabla_{x} w(x, t)-2 t w_{t}^{\prime}(x, t) \geqq d\left(y, K^{c}\right)(2 \pi t)^{-1 / 2} \exp \left[-\left(\Phi^{-1}(w(x, t))\right)^{2} / 2\right],
$$

where $d(x, y)=|x-y|=((x-y) \cdot(x-y))^{1 / 2}$ is the usual metric on $\mathbb{R}^{n}$ and

$$
\Phi(\lambda)=\int_{-\infty}^{\lambda} \exp \left(-s^{2} / 2\right) d s /(2 \pi)^{1 / 2}, \quad-\infty \leqq \lambda \leqq+\infty .
$$

Before the proof of (2.1) let us remark that the weaker differential inequality $(y-x) \cdot \nabla_{x} w(x, t)-t w_{t}^{\prime}(x, t) \geqq 0$ is a corollary to Theorem 1.1 .

In the following, let $\Omega$ be the standard Fréchet space of all continuous functions of $\left[0,+\infty\right.$ [ into $\mathbb{R}^{n}$ and assume $X-X(0)$ is represented as the identity mapping on $\Omega$. Stated otherwise, we choose the Wiener picture of Brownian motion. The isoperimetric inequality of Brownian motion may then be described as follows.

Suppose $U$ denotes the class of all absolutely continuous $\omega \in \Omega$ such that $\omega(0)=0$ and

$$
\int_{0}^{+\infty}\left|\omega^{\prime}(t)\right|^{2} d t \leqq 1
$$

Then $\Phi^{-1}\left(P_{x}[X \in A+\varepsilon U]\right) \geqq \Phi^{-1}\left(P_{x}[X \in A]\right)+\varepsilon, \varepsilon>0$, for each Borel set $A \subseteq \Omega$ (Borell [1]).

To prove (2.1) we may set $y=0$ and, of course, it suffices to treat the special case when 0 belongs to the interior of $K$. If $\bar{B}(0 ; r)$ denotes the closed ball in $\mathbb{R}^{n}$ of centre 0 and radius $r>0$, then $\omega([0, s]) \subseteq \bar{B}\left(0 ; s^{1 / 2}\right)$ for each $\left.(\omega, s) \in U \times\right] 0,+\infty[$, and, hence, for any fixed $\varepsilon>0$,

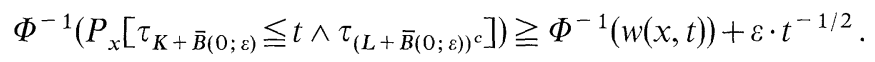

We now define $\varrho=1 / d\left(0 ; K^{c}\right)$ and have $M+\bar{B}(0 ; \varepsilon) \subseteq(1+\varepsilon \varrho) M, M=K, L$, because $K$ and $L$ are convex. Thus

$$
\Phi^{-1}\left(P_{x}\left[\tau_{(1+\varepsilon \varrho) K} \leqq t \wedge \tau_{((1+\varepsilon \varrho) L)^{c}}\right]\right) \geqq \Phi^{-1}(w(x, t))+\varepsilon \cdot t^{-1 / 2},
$$

and by scaling the time,

$$
\Phi^{-1}\left(w\left(x /(1+\varepsilon \varrho), t /(1+\varepsilon \varrho)^{2}\right) \geqq \Phi^{-1}(w(x, t))+\varepsilon \cdot t^{-1 / 2}\right.
$$

which immediately proves (2.1). 


\section{Step 2: Analysis of the Points of Discontinuity of $w$}

Assume $K$ satisfies the following additional conditions (iv) $K$ is strictly convex and (v) $K=K_{0}+\bar{B}\left(0 ; r_{0}\right)$, where $K_{0}$ is a convex body in $\mathbb{R}^{n}$ and $r_{0}>0$. Let $0<T<+\infty$ be fixed and set $D=\left\{(x, t) \in \mathbb{R}^{n} ; 0 \leqq t \leqq T\right\}, u=w_{\mid D}$, and

$$
\tilde{u}(\xi)=\sup \{u(\eta) \wedge u(\zeta) ; \xi \in[\eta, \zeta], \eta, \zeta \in D\}, \quad \xi \in D .
$$

respectively. Finally, suppose $\varepsilon \in] 0,1\left[\right.$ and let $\sup \left[\tilde{u}-u^{\varepsilon}\right]=Q>0$.

We claim there exist $\left.\left.\xi_{*}, \eta_{*}, \check{\zeta}_{*} \in(L \backslash K) \times\right] 0, T\right]$ such that $\tilde{u}\left(\xi_{*}\right)-u^{\varepsilon}\left(\xi_{*}\right)=Q$, $\left.\xi_{*} \in\right] \eta_{*}, \xi_{*}\left[\right.$, and $\tilde{u}\left(\xi_{*}\right)=u\left(\eta_{*}\right)=u\left(\zeta_{*}\right)$.

To see this, first note that the function $\tilde{u}-u^{\varepsilon}$ is non-positive on $\left\{\left(K \cup L^{c}\right) \times[0, T]\right\} \cup\{(L \backslash K) \times\{0\}\}$ and choose for each $i \in \mathbb{N}$ a $\left.\left.\xi_{i} \in(L \backslash K) \times\right] 0, T\right]$ with $q_{i}=\tilde{u}\left(\xi_{i}\right)-u^{\varepsilon}\left(\xi_{i}\right)>0$ and such that $q_{i} \rightarrow Q$ as $i \rightarrow+\infty$. Without any loss of generality we may assume the sequence $\left(\xi_{i}\right)_{i \in \mathbb{N}}$ converges to a point $\xi_{*} \in(\bar{L} \backslash \stackrel{K}{K}) \times[0, T]$. Next choose $\eta_{i}, \zeta_{i} \in D$ satisfying $\zeta_{i} \in\left[\eta_{i}, \zeta_{i}\right]$ and so that $0<u\left(\eta_{i}\right) \wedge u\left(\zeta_{i}\right)=\tilde{u}\left(\xi_{i}\right)-\delta_{i}$, where $0 \leqq \delta_{i} \rightarrow 0$ as $i \rightarrow+\infty$. If $\eta_{i} \in K \times[0, T]$, then by (2.1) the function $u\left(\zeta_{i}+\lambda\left(\eta_{i}-\zeta_{i}\right)\right), 0 \leqq \lambda \leqq 1$, increases and a similar assertion is true if $\zeta_{i} \in K \times[0, T]$. In view of these facts it may be assumed that $\left.\left.\eta_{i}, \zeta_{i} \in(L \backslash K) \times\right] 0, T\right]$. In the following $\hat{\eta}_{i}=\left(x\left(\hat{\eta}_{i}\right), t\left(\hat{\eta}_{i}\right)\right)$ denotes the point in $\left.\left.K \times\right] 0, T\right]$ which is closest to $\eta_{i}$ and we let $H\left(x\left(\hat{\eta}_{i}\right)\right)$ be the supporting hyperplane of $K$ at $x\left(\hat{\eta}_{i}\right)$. Analogous conventions will be used below with $\hat{\eta}_{i}$ replaced by $\hat{\zeta}_{i}$ and $\hat{\xi}_{i}$, respectively. Then, to begin with,

$$
u\left(\eta_{i}\right) \leqq P_{x\left(\eta_{i}\right)}\left[\tau_{H\left(x\left(\hat{\eta}_{2}\right)\right)} \leqq t\left(\eta_{i}\right)\right]
$$

that is $u\left(\eta_{i}\right) \leqq \Psi\left(d^{2}\left(x\left(\eta_{i}\right), K\right) / t\left(\eta_{i}\right)\right)$, where

$$
\Psi(\lambda)=\int_{0}^{1 / \lambda}\left(2 \pi s^{3}\right)^{-1 / 2} \exp (-1 /(2 s)) d s, \quad \lambda>0,
$$

and, in a similar way, $u\left(\zeta_{i}\right) \leqq \Psi\left(d^{2}\left(x\left(\zeta_{i}\right), K\right) / t\left(\zeta_{i}\right)\right)$. We now use that $\Psi$ decreases and that the function $\left.\left.d^{2}(x, K) / t,(x, t) \in \mathbb{R}^{n} \times\right] 0, T\right]$ is convex to obtain the inequality $u\left(\eta_{i}\right) \wedge u\left(\zeta_{i}\right) \leqq \Psi\left(\lambda_{i}\right)$, where $\lambda_{i}=d^{2}\left(x\left(\xi_{i}\right), K\right) / t\left(\xi_{i}\right)$. In particular,

$$
q_{i}-\delta_{i}+u\left(\xi_{i}\right) \leqq \Psi\left(\lambda_{i}\right)
$$

and, accordingly, the sequence $\left(\lambda_{i}\right)_{i \in \mathbb{N}}$ must be bounded. Now set $d\left(K, L^{c}\right)=R_{0}$ and choose $\bar{B}\left(y_{i} ; r_{0}\right) \cong K$ such that $x\left(\xi_{i}\right) \in \bar{B}\left(y_{i} ; r_{0}\right)$. Then

$$
u\left(\xi_{i}\right) \geqq P_{x\left(\xi_{i}\right)}\left[\tau_{\bar{B}\left(y_{i} ; r_{0}\right)} \leqq t\left(\xi_{i}\right) \wedge \tau_{B^{c}\left(y_{i} ; r_{0}+R_{0}\right)}\right]
$$

and introducing $\mu_{i}=r_{0} / t^{1 / 2}\left(\xi_{i}\right)$, this means that $u\left(\xi_{i}\right)$ does not fall below the probability

$$
P_{\left(\lambda_{1}^{1 / 2}, 0, \ldots, 0\right)}\left[\tau_{\mu_{1} \bar{B}((-1,0, \ldots, 0) ; 1)} \leqq 1 \wedge \tau_{\left(\mu_{i} B\left((-1,0, \ldots, 0) ; 1+R_{0} / r_{0}\right)\right)}\right] .
$$

Here, if $t\left(\xi_{*}\right)=0$, the same probability becomes arbitrarily close to $\Psi\left(\lambda_{i}\right)$ for large $i$, which contradicts (3.1). Thus $t\left(\xi_{*}\right)>0$.

From now on we assume without any loss of generality that the sequences $\left(\eta_{i}\right)_{i \in \mathbb{N}}$ and $\left(\zeta_{i}\right)_{i \in \mathbb{N}}$ both converge to the limits $\eta_{*} \in(\bar{L} \backslash \stackrel{\circ}{K}) \times[0, T]$ and $\zeta_{*} \in(\bar{L} \backslash \stackrel{\circ}{K})$ $\times[0, T]$, respectively. If $\eta_{*}$ or $\zeta_{*}=\xi_{*}$, the continuity of $u$ at $\xi_{*}$ implies the 
contradiction $u\left(\xi_{*}\right)-u^{\xi}\left(\xi_{*}\right) \geqq Q$. Hence $\left.\xi_{*} \in\right] \eta_{*}, \zeta_{*}\left[\right.$. If $x\left(\eta_{*}\right)=x\left(\zeta_{*}\right) \in K$, then $t\left(\eta_{*}\right)$ or $t\left(\zeta_{*}\right)>t\left(\xi_{*}\right)$, and by using the continuity of $u$ off $\partial K \times\{0\}$ we again obtain a contradiction. From these results and the strict convexity of $K$ it follows that $\eta_{*}$ or $\zeta_{*} \notin K \times[0, T]$. Assuming $\eta_{*} \notin K \times[0, T]$, we have $u\left(\eta_{*}\right)$ $-u^{\varepsilon}\left(\xi_{*}\right) \geqq Q$ and, in particular, $\left.\left.\eta_{*} \in(L \backslash K) \times\right] 0, T\right]$. If $\zeta_{*} \in K \times[0, T]$, then by (2.1) the function $u\left(\eta_{*}+\lambda\left(\zeta_{*}-\eta_{*}\right)\right), 0 \leqq \lambda \leqq 1$, increases and we get $u\left(\xi_{*}\right)$ $-u^{\varepsilon}\left(\xi_{*}\right) \geqq Q$, which is absurd. Consequently, $\zeta_{*} \notin K \times[0, T]$ and, as above, $u\left(\zeta_{*}\right)$ $-u^{\varepsilon}\left(\xi_{*}\right) \geqq Q$ and $\left.\left.\zeta_{*} \in(L \backslash K) \times\right] 0, T\right]$. From these facts, $\tilde{u}\left(\xi_{*}\right)-u^{\varepsilon}\left(\xi_{*}\right)=Q$, and by eventually moving $\eta_{*}$ or $\zeta_{*}$ closer to $\xi_{*}$ we have $u\left(\eta_{*}\right)=u\left(\zeta_{*}\right)=\tilde{u}\left(\xi_{*}\right)$, which completes the proof of the claim at the beginning of this section.

\section{Step 3: The Gabriel-Lewis Argument}

To prove Theorem 1.1 there is no loss of generality to assume that the conditions (i)-(v) are fulfilled. Let $u$ be as in the previous section. Of course, it is enough to show that the function $u$ is quasi-concave.

Suppose contrary to this that $u$ is not quasi-concave and choose an $\varepsilon \in] 0,1[$ with $\sup \left[\tilde{u}-u^{\varepsilon}\right]>0$. Let $\eta_{*}, \zeta_{*}$, and $\xi_{*}=\theta \eta_{*}+(1-\theta) \zeta_{*}$ be as in Step 2. This will lead us to a contradiction as follows.

First recall that $\nabla_{x} u \neq 0$ in $\left.\left.(L \backslash K) \times\right] 0, T\right]$ by (2.1) and suppose $h \in \mathbb{R}^{n}=\left(\mathbb{R}^{n} \times\{0\}\right)$ satisfies the inequality $h \cdot \nabla_{x} u\left(\eta_{*}\right)>0$. Then for all small $s>0, u\left(\eta_{*}+s h\right)>u\left(\eta_{*}\right)$ and, hence, $\tilde{u}\left(\xi_{*}+s \theta h\right) \geqq \tilde{u}\left(\xi_{*}\right)$ yielding $u\left(\xi_{*}+s \theta h\right) \geqq u\left(\xi_{*}\right)$ and $h \cdot \nabla_{x} u\left(\xi_{*}\right) \geqq 0$. From this follows that the vectors $\nabla_{x} u\left(\xi_{*}\right)$ and $\nabla_{x} u\left(\eta_{*}\right)$ are parallel and in the same way we conclude that the vectors $\nabla_{x} u\left(\xi_{*}\right)$ and $\nabla_{x} u\left(\zeta_{*}\right)$ are parallel.

Set $a=\left|\nabla_{x} u^{\varepsilon}\left(\xi_{*}\right)\right|, \quad b=\left|\nabla_{x} u\left(\eta_{*}\right)\right|, c=\left|\nabla_{x} u\left(\zeta_{*}\right)\right|$, and $v=\left(\nabla_{x} u^{\varepsilon}\left(\xi_{*}\right)\right) / a$, respectively. Suppose $h \in \mathbb{R}^{n}$ and $\kappa=h \cdot v \neq 0$. For each $s \in \mathbb{R}$ close to the origin there exists a unique $r=r(s)$ with $|r|$ minimal and such that $u\left(\eta_{*}+s h / b\right)=u\left(\zeta_{*}+r h / c\right)$. Writing $\xi_{s}$ $=\xi_{*}+(\theta s / b+(1-\theta) r(s) / c) h$, we now have $u\left(\eta_{*}+s h / b\right)-u^{\varepsilon}\left(\xi_{s}\right) \leqq u\left(\eta_{*}\right)-u^{\varepsilon}\left(\xi_{*}\right)$. In particular,

$$
\left\{\begin{array}{l}
D_{s}\left(u\left(\eta_{*}+s h / b\right)-u^{\varepsilon}\left(\xi_{s}\right)\right)_{\mid s=0}=0 \\
D_{s}^{2}\left(u\left(\eta_{*}+s h / b\right)-u^{\varepsilon}\left(\xi_{s}\right)\right)_{\mid s=0} \leqq 0
\end{array} .\right.
$$

Moreover, introducing

$$
u\left(\eta_{*}+s h / b\right)=u\left(\eta_{*}\right)+\kappa s+B s^{2}+\mathrm{o}\left(s^{2}\right) \quad \text { as } \quad s \rightarrow 0
$$

and

$$
u\left(\zeta_{*}+s h / c\right)=u\left(\zeta_{*}\right)+\kappa s+C s^{2}+o\left(s^{2}\right) \quad \text { as } \quad s \rightarrow 0
$$

it follows that

$$
r(s)=s+\kappa^{-1}(B-C) s^{2}+\mathrm{o}\left(s^{2}\right) \quad \text { as } \quad s \rightarrow 0 .
$$

By now setting $\lambda=\theta / b+(1-\theta) / c$ and

$$
u^{\varepsilon}\left(\xi_{*}+s h\right)=u^{\varepsilon}\left(\xi_{*}\right)+\kappa a s+A s^{2}+\mathrm{o}\left(s^{2}\right) \text { as } s \rightarrow 0,
$$

the above yields

$$
u^{\varepsilon}\left(\xi_{s}\right)=u^{\varepsilon}\left(\xi_{*}\right)+\lambda \kappa a s+\left[\lambda^{2} A+(1-\theta)(a / c)(B-C)\right] s^{2}+\mathrm{o}\left(s^{2}\right)
$$


as $s \rightarrow 0$. Thus from (4.1), $a=\lambda^{-1}$ and

$$
B-\left[\lambda^{2} A+(1-\theta)(a / c)(B-C)\right] \leqq 0 .
$$

To simplify the last inequality we define $\mu=\theta /(b \lambda)<1$ and so we have $\mu B$ $+(1-\mu) C-\lambda^{2} A \leqq 0$, that is

$$
\sum_{\substack{1 \leqq i \leqq n \\ 1 \leqq j \leqq n}}\left[\left(\mu / b^{2}\right) u_{x_{i} x_{j}}^{\prime \prime}\left(\eta_{*}\right)+\left((1-\mu) / c^{2}\right) u_{x_{i} x_{j}}^{\prime \prime}\left(\zeta_{*}\right)-\lambda^{2}\left(u^{\varepsilon}\right)_{x_{\iota} x_{j}}^{\prime \prime}\left(\xi_{*}\right)\right] h_{i} h_{j} \leqq 0 .
$$

Of course, the same estimate is true for all $h \in \mathbb{R}^{n}$ and, accordingly,

$$
\left(\mu / b^{2}\right) \Delta u\left(\eta_{*}\right)+\left((1-\mu) / c^{2}\right) \Delta u\left(\zeta_{*}\right)-\lambda^{2} \Delta u^{\varepsilon}\left(\xi_{*}\right) \leqq 0 .
$$

Since $\Delta u^{\varepsilon}\left(\xi_{*}\right)=2\left(u^{\varepsilon}\right)_{t}^{\prime}\left(\xi_{*}\right)+\varepsilon(\varepsilon-1) u^{\varepsilon-2}\left(\xi_{*}\right)\left|\nabla_{x} u\left(\xi_{*}\right)\right|^{2}$ and $\left|\nabla_{x} u\left(\xi_{*}\right)\right|>0$, necessarily

$$
\mu \beta / b^{2}+(1-\mu) \gamma / c^{2}-\lambda^{2} \alpha<0
$$

with $\alpha=\left(u^{\varepsilon}\right)_{t}^{\prime}\left(\xi_{*}\right)$, and where $\beta>u_{t}^{\prime}\left(\eta_{*}\right)$ and $\gamma>u_{t}^{\prime}\left(\zeta_{*}\right)$ are sufficiently small. But then

$$
1-\alpha(\theta / \beta+(1-\theta) / \gamma) \geqq 0,
$$

as the derivative

$$
D_{s}\left[u\left(\eta_{*}+(0, s / \beta)\right) \wedge u\left(\zeta_{*}+(0, s / \gamma)\right)-u^{\varepsilon}\left(\xi_{*}+(0, s(\theta / \beta+(1-\theta) / \gamma))\right)\right]_{\mid s=0^{-}}
$$

is non-negative. It is readily seen that (4.2) and (4.3) are non-consistent. In fact, by (4.3) the left-hand side of (4.2) does not fall below

$$
\begin{aligned}
& \mu \beta / b^{2}+(1-\mu) \gamma / c^{2}-\lambda^{2} /(\theta / \beta+(1-\theta) / \gamma) \\
& \quad=\lambda^{-1}(\theta / \beta+(1-\theta) / \gamma)^{-1}\left[\left(\theta \beta / b^{3}+(1-\theta) \gamma / c^{3}\right)(\theta / \beta+(1-\theta) / \gamma)-\lambda^{3}\right]
\end{aligned}
$$

where, by the Hölder inequality,

$$
\left(\theta \beta / b^{3}+(1-\theta) \gamma / c^{3}\right)(\theta / \beta+(1-\theta) / \gamma) \geqq\left(\theta / b^{3 / 2}+(1-\theta) / c^{3 / 2}\right)^{2} \geqq \lambda^{3} .
$$

From these estimates we have that the left-hand side member of (4.2) is nonnegative, which is a contradiction.

This completes the proof of Theorem 1.1.

\section{References}

1. Borell, C.: The Brunn-Minkowski inequality in Gauss space. Invent. math. 30, 207-216 (1975)

2. Friedman, A.: Stochastic differential equations and applications, Vol. 1. New York, San Francisco, London: Academic Press 1975

3. Gabriel, R.M.: An extended principle of the maximum for harmonic functions in 3-dimensions. $J$. London Math. Soc. 30, 388-401 (1955)

4. Gabriel, R.M.: A result concerning convex level surfaces of 3-dimensional harmonic functions. J. London Math. Soc. 32, 286-294 (1957)

5. Lewis, J.L.: Capacitary functions in convex rings. Arch. Rational Mech. Anal. 66, 201-224 (1977)

Communicated by B. Simon

Received February 16, 1982; in revised form April 16, 1982 
